\title{
Observation par fluorescence de la croissance du gamétophyte mâle dans le style et dans l'ovaire chez des hybrides Phaseolus coccineus $x$ Phaseolus acutifolius A. Gray et leurs allotétraploïdes
}

\author{
R. Ben Rejeb ${ }^{1}$ et A. Benbadis ${ }^{2}$ \\ 1 INRA, station de génétique et d'amélioration des plantes, centre de recherches agronomiques de Versailles, 78026 Versailles \\ Cedex; \\ 2 Laboratoire d'histophysiologie végétale, 12, rue Cuvier, 75005 Paris Cedex, France
}

(reçu le 4 novembre 1988, accepté le 21 avril 1989)

\begin{abstract}
Résumé - Deux combinaisons hybrides ont été obtenues entre $P$. coccineus et $P$. acutifolius par culture in vitro d'embryons immatures. Etant donnée la stérilité des hybrides $F_{1}$, tous les essais de croisements réalisés sur ces plantes ont été infructueux. Grâce à l'emploi de la microscopie en fluorescence, il a été possible d'observer la germination du pollen et de suivre la croissance des tubes polliniques dans le style et dans l'ovaire chez 2 hybrides $P$. coccineus $\mathrm{x}$ $P$. acutifolius après autopollinisation et rétrocroisement, et chez les allotétraploïdes correspondants après autopollinisation. Cette technique nous a permis de constater une croissance normale du gamétophyte mâle dans le style depuis la germination du grain de pollen sur le stigmate jusqu'à la pénétration du tube pollinique dans l'ovule. L'arrivée du tube pollinique au niveau de l'ovule a été observée $8 \mathrm{~h}$ après pollinisation chez les hybrides $\mathrm{F}_{1}$ et $14 \mathrm{~h}$ après autopollinisation chez les allotétraploïdes. La stérilité des hybrides $P$. coccineus $\times P$. acutifolius est le résultat de perturbation de nature physiologique intervenant durant ou après la fécondation en empêchant le développement de l'embryon.
\end{abstract}

microscopie en fluorescence - Phaseolus coccineus - Phaseolus acutifolius - hybride interspécifique - allotétraploïde - gamétophyte mâle - tube pollinique - incompatibilité

Summary - Fluorescence microscopy observation of male gametophyte growth in style and ovary of hybrids Phaseolus coccineus L x Phaseolus acutifolius A. Gray and of the corresponding allotetraploids. Two successful interspecific combinations were obtained with $\mathrm{P}$. coccineus and $\mathrm{P}$. acutifolius through embryo rescue. The $F_{1}$ hybrid plants were highly sterile and all cross attempts were conducted unsuccessfully. By using the technique of fluorescence microscopy it has been possible to observe the pollen germination and the growth of the pollen tubes in the style and in the ovary upon self - and cross - pollination of $\mathrm{P}$. coccineus $\times \mathrm{P}$. acutifolius hybrid plants and the corresponding allotetraploids after self-pollination. Normal growth of the male gametophyte in the style was observed from pollen grain germination until penetration of the pollen tube in the ovule. The pollen tubes reach the ovule 8 h after pollination for the $F_{1}$ hybrids and $14 \mathrm{~h}$ after sef-pollination for the allotetraploids. Sterility of $\mathrm{P}$. coccineus $\times \mathrm{P}$. acutifolius hybrids is due to physiological factors operating at, or after, the time of fertilization and preventing embryo development.

fluorescence microscopy - Phaseolus coccineus - Phaseolus acutifolius - interspecific hybrid - allotetraploid - male gametophyte - pollen tube - incompatibility

\section{INTRODUCTION}

Cette étude s'inscrit dans le cadre d'un programme de recherches sur les hybridations interspécifiques dans le genre Phaseolus. Ces travaux réalisés à la Station de génétique et d'amélioration des plantes de Versailles, visent essentiellement à identifier la nature des barrières d'incompatibilité entre les espèces croisées et les possibilités d'utilisation des hybrides interspécifiques pour l'amélioration des espèces cultivées du genre. Deux combinaisons hybrides ont été obtenues entre des lignées mâle stériles de $P$. coccineus $L$. et l'espèce $P$. acutifolius (génotype sauvage et génotype cultivé). La stérilité totale des iybrides $F_{1}$ a été vérifiée par des essais d'autopollinisation (manuelle) et de rétrocroisement par l'une ou l'autre forme parentale. Aucune graine mûre n'a été récoltée. Des nouaisons ont été fréquemment observées mais les gousses tombent avant d'at- 
teindre $2 \mathrm{~cm}$ de longueur. Cette autostérilité semble résulter principalement des perturbations occasionnées par l'association de 2 génomes très différents, car le traitement des plantules hybrides à la colchicine a permis de doubler le nombre chromosomique et de restaurer la fertilité de ces hybrides (Ben Rejeb \& Benbadis, 1988). Les plantes tétraploïdes ont montré une importante fertilité même si le nombre de graines par gousse est plus faible que chez les parents. Cependant le pourcentage de gousses arrivées à maturité $(26 \%)$ ne reflète pas le pourcentage de nouaison obtenu $(70 \%)$. En effet, de nombreuses gousses ont manifesté un arrêt de croissance en cours de développement.

L'objectif de la présente étude est de rechercher si l'apparente incompatibilité entre les génotypes parentaux est la conséquence directe de la croissance anormale des tubes polliniques dans le gynécée des fleurs pollinisées. Cette incompatibilité se traduit chez les hybrides $F_{1}$ par la chute prématurée des fleurs autopollinisées ou rétrocroisées ainsi que par l'avortement très précoce des gousses.

Hawkins \& Evans (1973), Tan (1979) ont montré que l'utilisation de la microscopie en lumière fluorescente est une technique efficace pour suivre la croissance du tube pollinique dans le style chez le genre Phaseolus. Cette méthode a été mise au point par Arens (1949) pour localiser la callose. Cette substance présente dans la paroi du tube pollinique ne possède pas de fluorescence propre. Au contact d'un certain nombre de fluorochromes tels que l'acridine orange ou le bleu d'aniline elle devient fluorescente (Stanley \& Linskens, 1974). Cette technique améliorée par Currier (1957), Martin (1959), Eschrich \& Currier (1964), a été reprise par plusieurs auteurs (Kho \& Baer, 1968; Chu Chou \& Harberd, 1970; Ramanna \& Mutsaerts, 1971; Gaget et al., 1984) pour suivre le cheminement des tubes polliniques chez plusieurs espèces.

\section{MATÉRIEL ET MÉTHODES}

Les observations ont été réalisées sur les hybrides interspécifiques résultant des 2 combinaisons suivantes: [Mo] $R_{1}(\mathrm{CT10}) \times \mathrm{N} 1700$ et CPD (1s) $\times$ NI901. [Mo] $R_{1}$ (CT10) et CPD (1s) sont 2 génotypes mâles stériles de $P$. coccineus le premier cytoplasmique, le second génique, utilisés comme parents femelles. Les 2 génotypes de $P$. acutifolius: NI700 et Ni901 employés comme parents mâles appartiennent respectivement à la forme sauvage et à la forme cultivée de cette espèce.

Le Tableau I donne l'origine et la provenance des génotypes parentaux choisis pour la réalisation des croisements.

Un grand nombre de fleurs ont été prélevées chez les hybrides $F_{1}$ après autopollinisation manuelle et
Tableau I. Génotypes parentaux utilisés dans les croisements entre $P$. coccineus (femelle) et $P$. acutifolius (mâle).

\begin{tabular}{|c|c|c|}
\hline Nom & Origine & Provenance \\
\hline \multicolumn{3}{|l|}{ P. coccineus } \\
\hline CPD (1s) & Mexique & $\begin{array}{l}\text { Matériel de } \\
\text { sélection }\end{array}$ \\
\hline$[\mathrm{Mo}] \mathrm{R}_{1}(\mathrm{CT} 10)$ & Mexique & H. Bannerot \\
\hline \multicolumn{3}{|l|}{ P. acutifolius } \\
\hline NI700 & Mexique & R. Maréchal \\
\hline NI901 & Salvador & R. Maréchal \\
\hline
\end{tabular}

CPD (1s) : Petaco (ms) $\times$ CT3

[Mo] : Cytoplasme de Morelos 662

après rétrocroisement par l'une ou l'autre espèce parentale, ainsi que chez les allotétraploïdes correspondants après autopollinisation. Les prélèvements ont été effectués à $10,12,14,16$ et 24 h après pollinisation. Le gynécée est fixé pendant $24 \mathrm{~h}$ dans une solution de Carnoy composée de : $100 \mathrm{ml}$ d'éthanol à $95 \%, 50 \mathrm{ml}$ de chloroforme pur et $16 \mathrm{ml}$ d'acide acétique glacial ( 20 gynécées fixés par prélèvement et par type de croissement). Ensuite ce matériel est mis à macèrer dans de la soude caustique $(8 \mathrm{~N})$ pendant 8 à $12 \mathrm{~h}$. Le rinçage à l'eau courante après macération permet de ramener le $\mathrm{pH}$ à $9, \mathrm{pH}$ optimum pour le traitement au bleu d'aniline.

Les styles (creux) sont en général séparés de l'ovaire du fait de leur différence de consistance, disséqués longitudinalement et leurs 2 faces internes sont disposées vers le haut pour recevoir la lumière incidente. Comme le style de Phaseolus est courbe, il est préférable de le découper préalablement en 2 ou 3 fragments cylindriques, ceux-ci sont ensuite fendus comme indiqué plus haut et examinés en présence du fluorochrome.

Les ovaires sont ouverts délicatement sur toute la ligne de suture du carpelle comme le préconise Tan (1979), de manière à maintenir les ovules en place sur le placenta. Les échantillons ainsi préparés sont prêts à recevoir le fluorochrome. La formule du réactif utilisé est la suivante: $\mathrm{Na}_{2} \mathrm{HPO} 4 \mathrm{0,05M}+0,05 \%$ de bleu d'aniline en poudre. La fluorescence apparaît au bout de quelques min. Le complexe callose-bleu d'aniline excité par la lumière ultra-violette émet une fluorescence jaune-vert (Eschrich \& Currier, 1964). Ce colorant est préconisé par Weilenmann de Tau et al. (1987) comme étant le meilleur fluorochrome de la callose à la suite de leurs observations sur l'hybride $P$. vulgaris $\times P$. filiformis.

Le microscope utilisé est un Leitz Dialux 20 à fluorescence en lumière UV (bloc de filtre : $A$, filtre d'excitation : BP 340-380, filtre d'arrêt : LP 430). Les photos ont été réalisées sur un film 400 ASA.

\section{RÉSULTATS}

\section{Observation chez les hybrides $F_{1}$}

Des croisements manuels ont été réalisés sur les plantes hybrides $F_{1}$ issues des 2 combinaisons 
[Mo] $R_{1}(C T 10) \times$ NI700 et CPD (1s) $\times$ NI901 (Tableau II). Si quelques jeunes gousses ont pu se développer sur ces hybrides, soit après autopollinisation, soit après rétrocroisement par l'un ou l'autre parent, aucune gousse ne persiste audelà de $5 \mathrm{j}$. Chez les 2 combinaisons hybrides, l'observation en fluorescence du stigmate montre un nombre très faible de grains de pollen qui germent après autopollinisation (Fig. 1). A la surface du stigmate, les grains de pollen restent associés en tétrades et n'émettent pas de tubes polliniques. Ces observations viennent confirmer les résultats des tests d'analyse de fertilité du pollen des hybrides par culture in vitro ou par coloration au carmin acétique de Belling. Ces tests ont montré que la germination du pollen in vitro dans un milieu approprié et sous conditions contrôlées (Lin et Dickinson, 1984) est très faible $(<10 \%)$ par rapport au pollen du témoin fertile, $P$. acutifolius, qui germe à $80 \%$ dans les mêmes conditions de culture. Le test classique de coloration

Tableau II. Taux de réussite des pollinisations réalisées chez les hybrides $P$. coccineus $\times P$. acutifolius.

Types de croisement $\quad P \quad N \quad \% N \quad R$

[Mo] $R_{1}(\mathrm{CT} 10) \times \mathrm{N} 1700$

Rétrocroisements :

hybride $F 1 \times P$. coccineus $186 \quad 120 \quad 64,5 \quad 0$

hybride F1 $\times$ P. acutifolius $\quad 96 \quad 46 \quad 48,0 \quad 0$

$\begin{array}{lrrrr}\text { Autofécondation : } & 102 & 0 & 0,0 & 0\end{array}$

CPD (1s) $\times$ N1901

Rétrocroisements :

hybride $F 1 \times P$. coccineus $\quad 86 \quad 45 \quad 52,3 \quad 0$

hybride F1 $\times$ P. acutifolius $\quad \begin{array}{rrrr}105 & 72 & 68,5 & 0\end{array}$

$\begin{array}{lllll}\text { Autofécondations : } & 70 & 9 & 12,8 & 0\end{array}$

$P$ : nombre de fleurs pollinisées

$\mathrm{N}$ : nombre de fleurs nouées

$\mathrm{R}$ : nombre de gousses récoltées

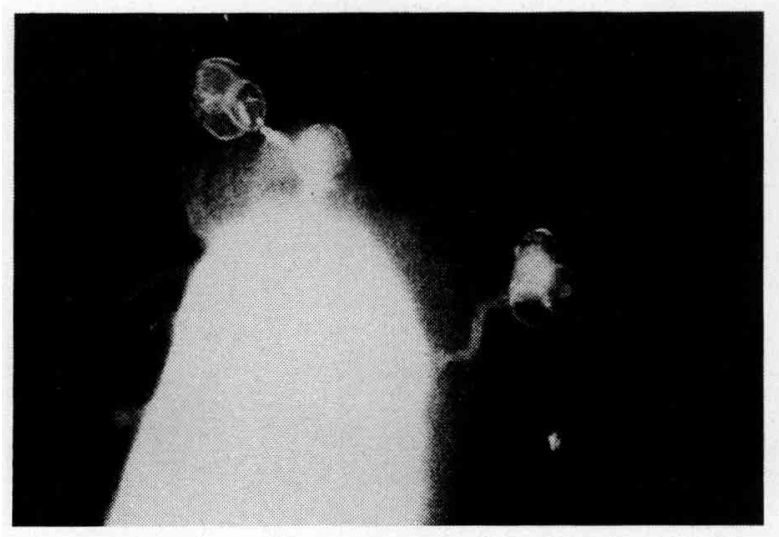

Fig. 1. Germination du pollen sur le stigmate après autopollinisation chez l'hybride P. coccineus x P. acutifolius (x 100). du pollen au carmin acétique a montré un pourcentage très faible de pollen régulièrement formé et coloré chez les hybrides $F_{1}(27 \%$ en moyenne). Les grains de pollen sont souvent associés en tétrade avec un grand nombre de grains vides de très faible diamètre ou, à exine normalement constituée, mais ne contenant que des traces de cytoplasme. Le pollen du témoin fertile est, à $98 \%$, de forme régulière, non aggloméré et coloré. L'observation en fluorescence du style montre la présence de quelques rares tubes polliniques.

Après autopollinisation abondante chez les hybrides $F_{1}$ avec le pollen provenant d'au moins 2 fleurs de la même plante, il a cependant été possible d'avoir un grand nombre de grains de pollen qui germent à la surface du stigmate et de suivre l'évolution du tube pollinique dans la partie creuse du style, puis dans l'ovaire.

En rétrocroisement, il a été facile de suivre le cheminement des tubes polliniques compte tenu du nombre assez élevé de grains de pollen qui germent sur le stigmate (Fig. 2). Dans le style, la croissance des tubes polliniques étant très rapide, le dépôt de callose responsable de leur fluorescence n'est plus suffisant pour les rendre visibles. Ils se confondent ainsi avec les tissus du style, ce qui rend leur identification difficile. Cette difficulté a été signalée également par Hawkins \& Evans (1973) chez P. vulgaris L. et par Tan (1979) chez $P$. lunatus L. Néanmoins nous avons pu observer quelques tubes polliniques dans la cavité centrale du style (Fig. 3).

L'entrée du tube pollinique dans l'ovaire s'accompagne d'un ralentissement de sa vitesse de croissance, donc d'un fort dépôt callosique. La fluorescence du tube pollinique réapparaît donc dans l'ovaire (Fig. 4). La Figure 5 montre un tube pollinique qui pénètre l'ovule par le micropyle (porogamie). Ces observations ont été faites sur les échantillons prélevés $8 \mathrm{~h}$ après pollinisation (aussi bien après autopollinisation qu'après rétro-

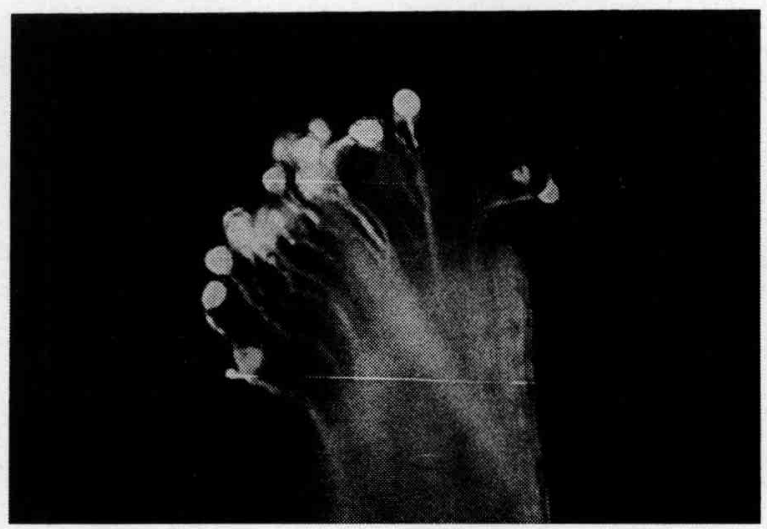

Fig. 2. Germination du pollen sur le stigmate après rétrocroisement de l'hybride $P$. coccineus $\times P$. acutifolius par le parent $P$. acutifolius . 


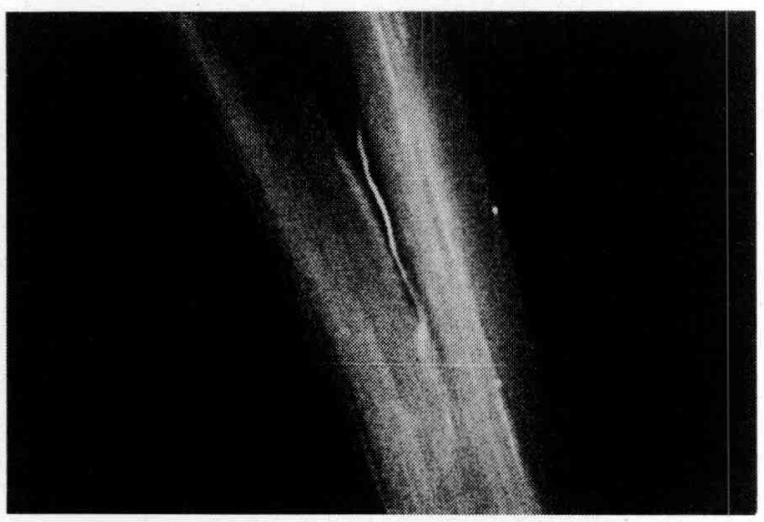

Fig. 3. Tube pollinique dans la partie médiane du style (x 100).

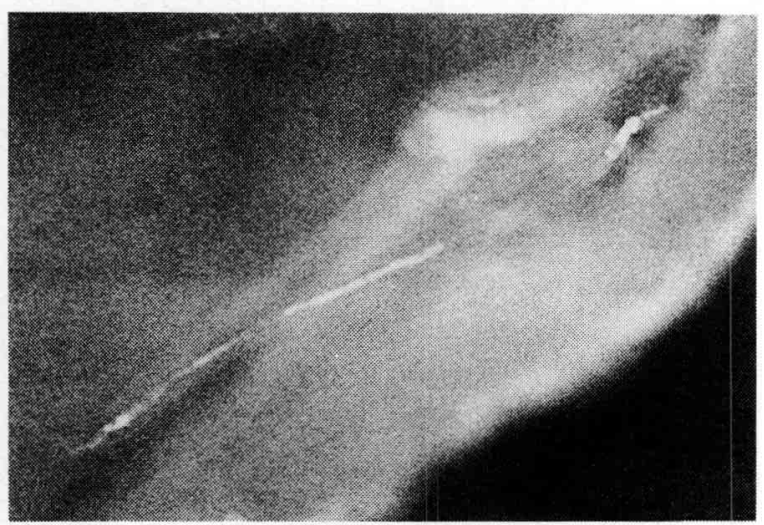

Fig. 4. Tube pollinique dans l'ovaire $(\times 100)$.

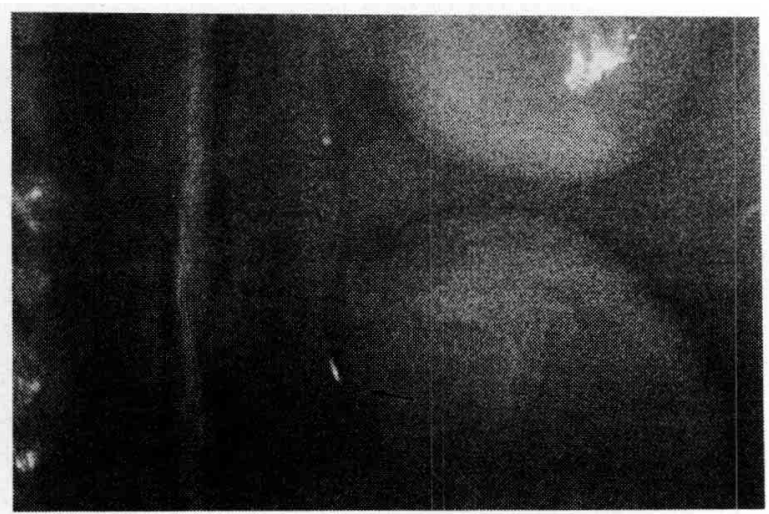

Fig. 5. Le tube pollinique pénètre dans l'ovule par le micropyle $(x 100)$.

croisement). Cette constatation concorde bien avec les résultats de Weinstein (1926) chez $P$. vulgaris et Tan (1979) chez $P$. lunatus, qui ont montré que les premiers tubes polliniques atteingent les ovules dans les $8 \mathrm{~h}$ après pollinisation pour des styles de 12 à $15 \mathrm{~mm}$.

\section{Observations chez les allotétraploïdes}

La restauration de l'autofertilité chez les hybrides $F_{1}$ doublés est marquée par le nombre très élevé de grains de pollen qui germent sur le stigmate (Fig. 6) $(81,5 \%$ des grains de pollen sont colorés chez les allotétraploïdes lors de tests au carmin acétique de Belling. Le repérage du tube pollinique dans la partie médiane du style se fait sans difficulté. II a même été possible de suivre son trajet sur une longue distance à partir du stigmate (Fig. 7). II est important de remarquer que chez l'allotétraploüde, l'arrivée du tube pollinique au niveau de l'ovule a été observée uniquement pour les échantillons prélevés $14 \mathrm{~h}$ après autopollinisation. Tous les échantillons prélevés après 8 ou $12 \mathrm{~h}$ ont montré seulement des parties fluorescentes de tubes polliniques au début ou au milieu du style et rarement à l'entrée de l'ovaire. Weilenmann de Tau et al. (1987) ont constaté également la pénétration de l'ovule par le tube pollinique dans des échantillons prélevés $18 \mathrm{~h}$ après rétrocroisement de l'allotétraplö̈de $P$. vulgaris $\times P$. filiformis par le parent $P$. vulgaris.

A l'entrée de l'ovule, le tube pollinique forme un coude et s'introduit dans le micropyle en formant parfois une boursouflure à ce niveau (Fig. $8, a, b)$. Certaines préparations montrent une pénétration du tube à travers les téguments dans des régions voisinant le micropyle (Fig. 9).

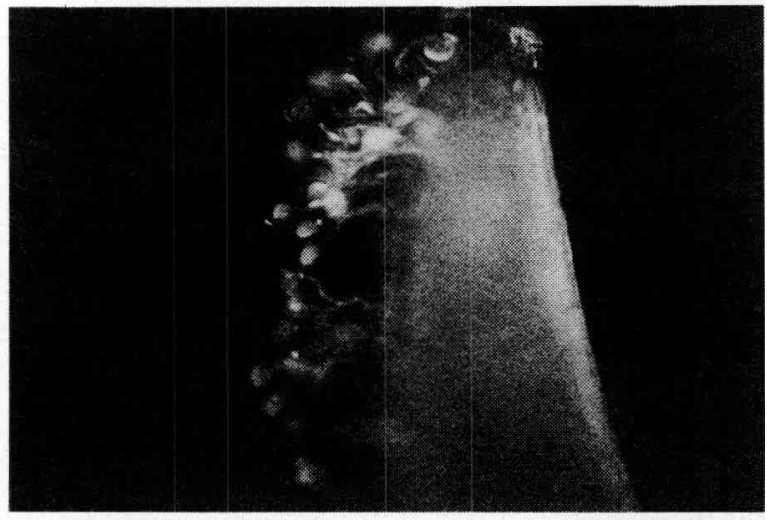

Fig. 6. Germination du pollen sur le stigmate après autopollinisation chez l'allotétraploïde $P$. coccineus $\times P$. acutifolius (x 100).

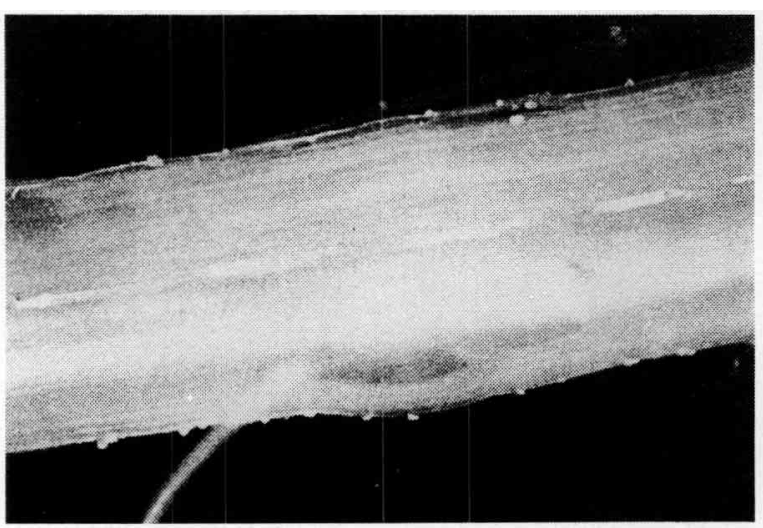

Fig. 7. Tube pollinique observé sur une longue distance dans le style chez l'allotétraploìde (x 100). 
(a)

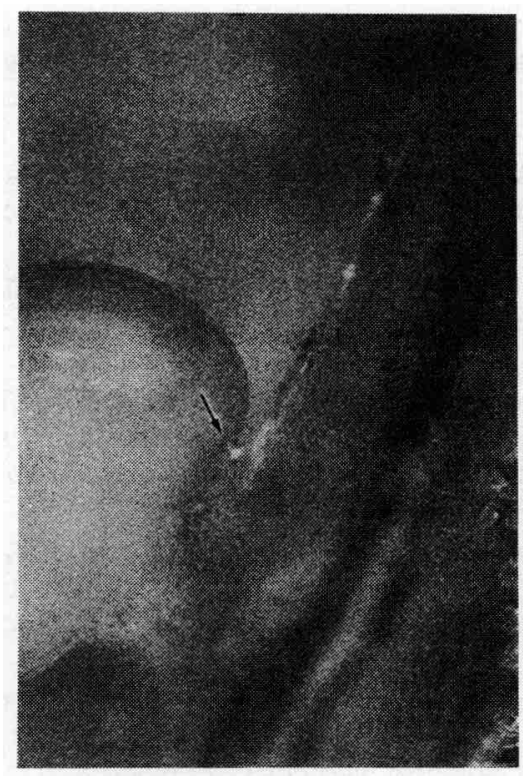

Fig. 8. Pénétration de l'ovule par le tube pollinique (x 100).

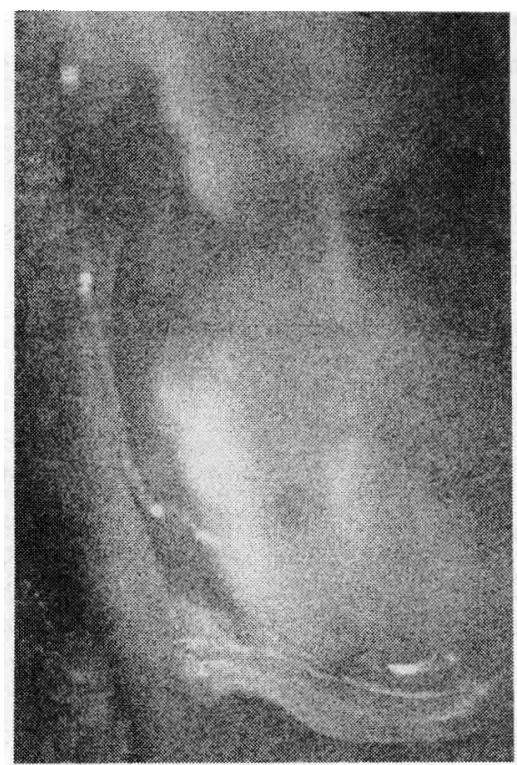

Fig. 9. Tube pollinique déplacé de sa position initiale $(\times 100)$.

L'examen minutieux de ces cas a montré que lors des manipulations, les ovules peuvent subir des perturbations qui entraînent le déplacement du tube pollinique de sa position initiale. II est donc impératif de respecter la structure initiale des tissus. Des constatations similaires ont été rapportées par Tan (1980).

\section{DISCUSSION ET CONCLUSION}

Cette méthode d'observation par fluorescence nous a permis d'observer la germination du pollen sur le stigmate, de suivre son trajet dans la cavité creuse du style (lorsque le dépôt de callo- (b)

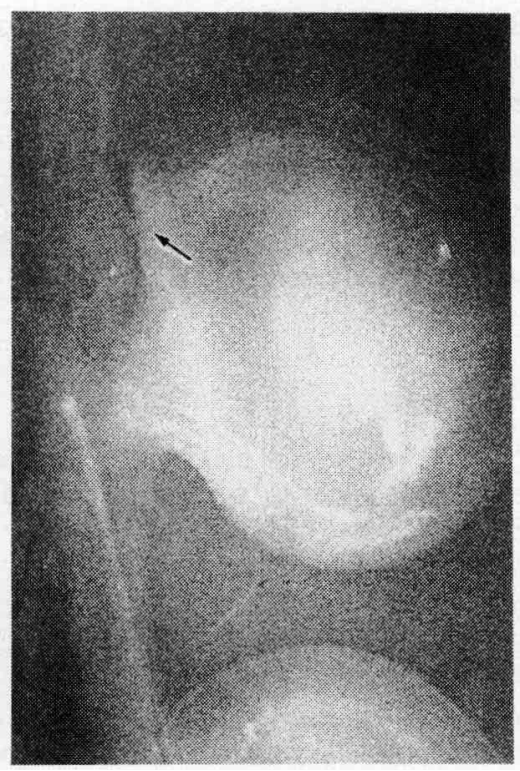

se est suffisant) puis dans l'ovaire et sa pénétration dans l'ovule. Les résultats obtenus montrent une croissance normale du gamétophyte mâle aussi bien chez les hybrides $F_{1}$ que chez les allotétraploïdes correspondants avec cependant une différence concernant le temps mis par le tube pollinique pour atteindre l'ovule: $8 \mathrm{~h}$ pour l'hybride et $14 \mathrm{~h}$ pour l'allotétraploïde. La croissance lente des tubes polliniques chez l'allotétraploïde peut être due à la différence de ploïdie. Chez l'hybride $F_{1}$, le nombre très faible de grains de pollen qui germent sur le stigmate est le résultat d'une méiose asyndétique (3,2 univalents en moyenne pour les 2 combinaisons) aboutissant à un faible pourcentage de pollen fertile. Ces observations montrent que l'incompatibilité n'est pas due à une croissance anormale du gamétophyte mâle. Dans le genre Phaseolus ainsi que chez les autres légumineuses à grosses graines (Vicia faba) (Ramsay \& Pickergill, 1986), les principales difficultés rencontrées dans la réalisation des hybrides interspécifiques varient dans leur nature en fonction des espèces utilisées mais surviennent généralement après le processus de fécondation. L'hypothèse d'une incompatibilité entre l'embryon et les tissus maternels est souvent discutée. Le Marchand et al. (1976), Rabakoarihanta et al. $(1979,1980)$ ont montré des retards dans l'initiation de l'albumen ou dans les divisions embryonnaires qui se traduisent par une malformation de l'embryon ou par l'absence d'albumen normalement développé. II serait intéressant dans le cas de l'hybride $P$. coccineus $x$ $P$. acutifolius de suivre la double fécondation, les événements qui la précèdent puis les phases de l'embryogenèse par la technique d'éclaircissage de Herr (Levieil \& Huyghe, 1985). 


\section{REMERCIEMENTS}

Nous tenons à remercier M. Hubert Bannerot pour ses précieux conseils, ses critiques constructives et pour la correction du manuscrit. Nous remercions également $M$. Jacques Schmit et le personnel du laboratoire de Bactériologie (INRA, Versailles) où nous avons pu réaliser l'étude microscopique.

\section{RÉFÉRENCES}

Arens K. (1949) Provo de callose por meio da microscopia a luz fluorescente e applicaçoes do metodo. Lilloa. 18, 71-75

Ben Rejeb R. \& Benbadis A. (1988) Fertile allotetraploid from the cross between Phaseolus coccineus $L$. and Phaseolus acutifolius A. Gray. Plant Cell Rep. (sous presse)

Chu Chou M. \& Harberd D.J. (1970) Note on visual distinction of fluorescent callose of pollen tubes and of sieve tubes in stylar tissue of Brassica and its allies. Euphytica 19, 379-381

Currier H.B. (1957) Callose substance in plant cells. Am. J. Bot. 44, 478-488

Eschrich W. \& Currier H.B. (1964) Identification of callose by its diachrome and fluorochrome reaction. Stain Technol. 39, 303-308

Gaget M., Saïd C. \& Dumas C. (1984) Pollen-pistil interactions in interspecific crosses of Populus (sections aigeiros and leuce) : pollen adhesion, hydration and callose reponses. J. Cell. Sci. 72, 173-184

Hawkins C.F. \& Evans A.M. (1973) Elucidating the behaviour of pollen tubes in intra- and interspecific pollinations of Phaseolus vulgaris $L$. and $P$. coccineus Lam. Euphytica 22, 378-385

Kho Y.O. \& Baer J. (1968) Observing pollen tubes by means of fluorescence. Euphytica 17, 298-302

Le Marchand G., Maréchal R. \& Baudet J.C. (1976) Observation sur quelques hybrides dans le genre Phaseolus. III. P. lunatus : nouveaux hybrides et considérations sur les affinités interspécifiques. Bull. Rech. Agron. Gembloux. 11, 183-199

Levieil C. \& Huyghe C. (1985) Observation des gamétogenèses mâle et femelle, de la fécondation et de la formation d'embryons non zygotiques après éclaircissage des anthères et sacs embryonnaires de Cichorium intybus L. et de Linum usitatissimum L., $C$. $R$. Acad. Sci. Paris 303, ser. 3, 7, 373-378

Lin J.J. \& Dickinson D.B. (1984) Ability of pollen to germinate prior to anthesis and effect of desiccation on germination. Plant Physiol. 74, 746-748

Martin F.W. (1959) Staining and observing pollen tubes by means of fluorescence. Stains Technol. 34, 125128

Rabakoarihanta A., Mok D.W.S. \& Mok C. (1979) Fertilization and early embryo development in reciproca interspecific crosses of Phaseolus. Theor Appl. Genet. 54, 55-59

Rabakoarihanta A., Schii C.T., Mok M.C. \& Mok C (1980) Meiosis and fertility of interspecific hybrids between Phaseolus vulgaris $L$. and Phaseolus acutifolius A. Gray. Theor. Appli. Genet. 57, 2, 59-64

Ramanna M.S. \& Mutsaerts M.C.A. (1971) Usual behaviour of growing pollen tubes in the styles and ovules of Spinacia oleracea L. Euphytica 20, 145-151

Ramsay G. \& Pickersgill B. (1986) Interspecific hybridization between Vicia faba and other species of Vicia: approches to delaying embryo abortion. Biol. Zentral. b1 $105,171-179$

Stanley R.G. \& Linskens H.F. (1974) Pollen. SpringerVerlag Berlin Heidelberg New-York

Tan B.S. (1979) Observation par fluorescence de la croissance du gamétophyte mâle chez Phaseolus lunatus $L$. et chez un hybride interspécifique. Bull. Rech. Agron. Gembloux 14, 97-108

Tan B.S. (1980) Recherche d'une méthode permettant d'observer le développement du gamétophyte mâle dans le gynécée de Phaseolus lunatus $L$. avec une application dans le cas d'un hybride interspecifique. Thèse Univer. Franche-Comté, Nord. 50

Weilenmann de Tau E., Mathieu A., Maréchal R. \& Baudoin J.P. (1987) Observation de la croissance du gamétophyte mâle chez des allotétraploïdes d'un hybride interspécifique entre Phaseolus vulgaris L. et Phaseolus filiformis Benth. Bull. Rech. Agron. Gembloux 22, 143-151

Weinstein A.I. (1926) Cytological studies on Phaseolus vulgaris. Am. J. Bot. 13, 248-263 